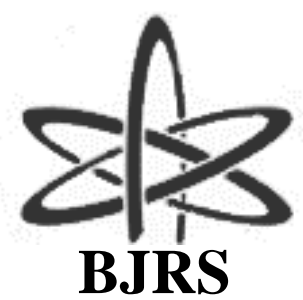

BRAZILIAN JOURNAL

$\mathrm{OF}$

RADIATION SCIENCES

03-01 (2015) 01-10

\title{
Recomendações para a garantia da segurança em um setor de ressonância magnética
}

\author{
T. J. Jormada ${ }^{\mathrm{a}}$; R. B. Medeiros ${ }^{\mathrm{b}}$ \\ ${ }^{a}$ Departamento de Diagnóstico por Imagem e Setor de Física e Higiene das Radiações(DDI)/Universidade Federal \\ de São Paulo/Escola paulista de Medicina (Unifesp/EPM), \\ 04044-010, São Paulo-SP, Brasil \\ ${ }^{b}$ Departamento de Diagnóstico por Imagem (DDI)/Universidade Federal de São Paulo (Unifesp/EPM), \\ 04021-002, São Paulo-SP, Brasil \\ tiagosjornada@gmail.com
}

\section{RESUMO}

No mês de setembro de 2014 três acidentes foram relatados, envolvendo pacientes e funcionários em ambientes de Imagens por Ressônancia Magnética (MRI). Órgãos internacionais como o American College of Radiology (ACR), The Emergency Care Research Institute (ECRI), Institute for Magnetic Resonance Safety, Education, and Research (IMRSER) e o Food and Drug Administration (FDA) estabelecem requisitos de segurança, que que podem ser adotados nos países sem uma legislação especifica na área, como no caso do Brasil. Sendo assim o objetivo do presente trabalho é divulgar as recomendações quanto à segurança em uma instalação que contemple um ou mais equipamentos de RM. Dentre as normativas específicas destacam-se as recomendações do ECRI quanto à política de fiscalização, ressaltando a necessidade de se criar oficialmente o cargo de segurança ou responsável por garantir que os procedimentos ou as normativas sejam aplicadas e atualizadas. Sendo que é consenso entre os órgãos, considerando a orientação de que a segurança precisa ser reforçada, evidencia-se a necessidade de um profissional capacitado para fiscalizar, orientar e oferecer treinamento contínuo a todos os profissionais envolvidos na aquisição de imagens por ressonância magnética.

Palavras-chave: Ressonância Magnética, Segurança, Normativas, Acidentes. 


\section{INTRODUÇÃO}

Uma alternativa ao uso das radiações ionizantes é a obtenção de Imagens por Ressonância Magnética (MRI do inglês Magnetic Resonance Imaging). As inúmeras aplicações desta técnica, vêm consolidando-a como o padrão ouro no campo diagnóstico, fizeram a quantidade de aparelhos de ressonância magnética crescer consideravelmente; em 1999 a sudeste possuía apenas 176 aparelhos instalados segundo o Instituto Brasileiro de Geografia e Estatística (IBGE, 1999) [1], já no ano de 2013 somente o estado de São Paulo já contava com 357 aparelhos em pleno funcionamento (CNES, 2013) [2]. Como consequência direta ao aumento na oferta de equipamentos de MRI, a demanda na realização de exames região aumentou. Tendo em vista que a finalidade de uma imagem diagnóstica é fornecer ao médico informações suficientes para um laudo confiável e preciso, assegurando sempre a integridade física do paciente, e mesmo sendo considerada por muitos uma técnica segura, não há estudos suficientes que garantam que o campo magnético gerado não irá causar algum tipo de detrimento ao paciente.

Segundo o Food and Drug Administration (FDA), nos Estados Unidos a taxa de acidentes entre os anos de 2000 e 2009 aumentou 500\%, enquanto o número de exames cresceu 90\% no mesmo período. Entre os acidentes mais comuns estão as lesões por "projétil", na qual um objeto ferromagnético é atraído em direção ao magneto em alta velocidade. O incidente deste tipo muito divulgado ocorreu em 2001, quando um menino de 6 anos morreu depois que um tanque de oxigênio voou pelo ar a 30 metros por segundo, fraturando o crânio da criança [3]. Há também relato de casos de reações adversas agudas devido ao uso de agente de contraste, como laringoespasmo e choque anafilático. Nestes casos, a taxa de incidência total varia entre $2 \%$ a $4 \%$ [4].

Abaixo constam alguns incidentes relatados pelo FDA [4].

- Um paciente com um marca-passo cardíaco implantado morreu durante um exame de RM. (12/2/92).

- $\quad$ Um paciente com um marca-passo cardíaco implantado morreu durante ou logo após um exame de RM. O legista determinou que a morte foi causada pela interrupção do marca-passo pelo sistema de MRI. (9/18/89). 
- Um paciente com um clipe de aneurisma intracraniano implantado morreu por causa de um exame de MRI. O clipe deslocou-se quando foi exposto ao campo magnético. A equipe aparentemente havia obtido informações indicando que o material neste clipe poderia ser utilizado de forma segura. (11/11/92).

- Deslocamento de um depósito de ferro no olho de um paciente durante a ressonância magnética resultou em perda de visão. (1/8/85).

- $\quad$ O paciente queixava-se de visão dupla após uma MRI. Ele foi submetido a uma MRI e a um exame raios-X, e revelou a presença de metal perto do seu olho. O paciente foi sedado no momento do exame, e não foi capaz de informar sobre a procedência do material metálico. (12/15/93).

- Um objeto foi atraído para o ímã e atingiu um paciente, cortando seu braço. O paciente necessitou de grampeamento do corte. (8/30/94).

- Um par de tesouras foi puxado das mãos de um enfermeiro, quando ele entrou na sala de MRI. A tesoura atingiu o paciente causando um corte na cabeça dele. (8/2/93).

- Um paciente foi atingido por uma garrafa de oxigênio enquanto ele posicionado dentro do gantry. Ele sofreu ferimentos com necessidade de pontos. (6/2/91).

- Dois dentes de aço (partes de uma empilhadeira), pesando 80 quilos cada, foram acelerados pelo ímã acertando um técnico e derrubando-o, resultando em ferimentos graves. $(6 / 5 / 86)$.

- Um fio eletricamente condutivo foi enrolado e colocado contra a pele, causando uma queimadura na parte superior do braço do paciente. (5/19/95)

- Uma criança teve uma queimadura na mão direita provocada por um cabo de ECG, enquanto era anestesiado. Um enxerto de pele foi necessário para tratar a área afetada. (1/26/95).

- Um paciente com uma bomba de infusão de insulina foi colocado em um ambiente de ressonância magnética, o que causou na movimentação do dispositivo. A bomba foi removida do paciente e posteriormente verificou-se que o dispositivo não era compatível ao ambiente $(1 / 13 / 88)$.

No mês de setembro 2014, na Índia, um operador foi atingido por um cilindro de oxigênio que o prensou contra a Gantry, resultando em ferimentos graves. No mesmo mês outros dois acidentes foram relatados, um na Nova Zelândia, no qual um operador foi atingido por uma faca que 
estava de posse do paciente; outro, mais uma vez na Índia, onde uma paciente sofreu ferimentos quando uma maca foi introduzida na sala [5-7].

\section{MATERIAIS E MÉTODOS}

Órgãos internacionais como o American College of Radiology (ACR), The Emergency Care Research Institute (ECRI), Institute for Magnetic Resonance Safety, Education, and Research (IMRSER) e o Food and Drug Administration (FDA) estabelecem requisitos de segurança para um ambiente de imagens por ressonância magnética. Sendo assim, o objetivo do presente trabalho é divulgar as recomendações de segurança em uma instalação que contemple um ou mais equipamentos de MRI.

\section{RESULTADOS E DISCUSSÕES}

O IMSER [8] enfatiza que adotar procedimentos de triagem completos e eficazes para os pacientes e outras pessoas é um dos componentes mais críticos. Um aspecto importante para proteger os pacientes e indivíduos de acidentes ou lesões relacionadas ao sistema de MRI envolve uma compreensão dos riscos associados aos implantes, aparelhos, acessórios ou outros objetos ferromagnéticos. Tais objetos requerem atenção constante e diligência de poder para obter informações e documentações sobre eles. Além disso, como a maioria dos incidentes relacionados com à RM tem sido causada por deficiências nos métodos de triagem e / ou por falta de controle adequado ao acesso no ambiente de MRI (especialmente no que diz respeito à prevenção de itens pessoais e outros objetos potencialmente problemáticos dentro numa sala de sistema de IRM).

Certos aspectos da triagem de pacientes para procedimentos de MRI podem ocorrer durante 0 processo de agendamento. Essas atividades devem ser realizadas por um profissional de saúde devidamente qualificado em segurança de MRI, ou seja, o profissional deve estar apto/treinado para entender os riscos potenciais e os problemas associados ao ambiente, para realizar os procedimentos e estar familiarizado com as informações contidas nos formulários de triagem para os pacientes e indivíduos. Durante o agendamento deve-se questionar se o paciente se ele possui um implante ou um dispositivo que possa ser contraindicado ou necessite uma especial atenção para a 
realização do procedimento: por exemplo, um grampo de aneurisma ferromagnético, marca-passo, ou sistema de neuroestimulação, dentre outros. Além disso, é necessário avaliar se há indicação que necessita de uma análise cuidadosa, por exemplo, com pacientes grávidas, pessoas com necessidades especiais, história de insuficiência renal, corpo estranho metálico no corpo, dentre outros. A triagem preliminar ajuda a impedir que os pacientes agendados possam ser candidatos inapropriados para exames de MRI.

Após a triagem preliminar, o paciente deve passar por uma triagem mais abrangente, para preparação e realização do procedimento de MRI. Essa triagem abrangente envolve o uso de um formulário impresso para documentar o processo de rastreio, uma revisão das informações no formulário de triagem e uma entrevista verbal para verificar as informações. Um profissional de saúde treinado em segurança de MRI deverá conduzir esses tais procedimentos.

Questões relativas à data do último período menstrual, uso de contraceptivos orais ou terapia hormonal e medicamentos de fertilidade são necessárias para pacientes do sexo feminino submetidas a procedimentos de MRI para avaliar doenças da mama ou para aplicações de OB / GYN, já que estes podem alterar o contraste de tecidos em ressonância magnética. Deve-se obter dados no caso da administração de meios de contraste em mães em processo de amamentação.

Com o uso de qualquer tipo de questionário escrito, existem limitações relacionadas a respostas incompletas ou incorretas fornecidas pelo paciente. Por exemplo, pode haver dificuldades em caso de pacientes que tem a visão prejudicada, dificuldade na fluência do idioma, ou baixo nível de alfabetização. Portanto, um membro da família que acompanha ou outra pessoa devidamente capacitada devem ser envolvidos no processo de triagem para verificar qualquer informação que possa afetar a segurança do paciente. Versões deste formulário também devem estar disponíveis em outros idiomas.

No caso em que o paciente está em coma ou incapaz de se comunicar, a forma de rastreio escrito deve ser concluída pelo indivíduo mais qualificado (por exemplo, médico, membro da família ou outro) sobre o histórico médico do paciente e condição atual. Se as informações de rastreio são insuficientes, é aconselhável procurar cicatrizes cirúrgicas no paciente e / ou realizar radiografias simples do crânio e / ou peito em busca de implantes que possam ser perigosos no ambiente de MRI (por exemplo, clipes de aneurisma, marca-passo, sistemas de neuroestimulação, etc.) 
Após o procedimento de triagem abrangente for concluído o paciente deve ser transferido por uma maca ou cadeira de rodas para a sala de sistema de MRI, tais equipamentos de transporte devem ser verificados cuidadosamente para não conter objetos metálicos, como tanques de oxigênio ferromagnético, monitores ou outros objetos que possam representar perigo.

O uso adequado desta forma escrita do formulário de triagem, juntamente com a triagem verbal completa do indivíduo, por um segurança de MRI, evitará acidentes e lesões no ambiente de realização dos exames.

O ECRI e o FDA [9] recomendam nomear um oficial de segurança em MRI ou outra pessoa responsável por garantir que os procedimentos apropriados estejam em vigor, aplicados, e atualizados para garantir a segurança no ambiente de MRI. Estabelecer rotinas para rever as políticas e procedimentos de segurança de ressonância magnética, e avaliar o grau de cumprimento por todos os membros da equipe. Fornecer a todos os envolvidos nos procedimentos de imagens por ressonância magnética, juntamente com outros funcionários que teriam uma oportunidade ou necessidade de entrar no ambiente MR (por exemplo, o pessoal de transporte, agentes de segurança, pessoal de limpeza, trabalhadores de manutenção, pessoal do departamento de incêndio, etc) treinamento em segurança da ressonância magnética. Isso deve ser feito especialmente para os novos empregados e repetido em uma base regular, isto é, anualmente. Compreender e enfatizar a todo o pessoal que o campo magnético estático do sistema de RM está sempre ligado.

Não permitir que equipamentos e dispositivos que contenham componentes ferromagnéticos adentrem o ambiente de MRI, a menos que tenham sido testados e rotulados de Seguro a RM.

Seguir quaisquer restrições fornecidas por fornecedores quanto ao uso de equipamentos e dispositivos Seguro a MRI e / ou Compatível a MRI. Um rótulo de MRI Seguro significa que o dispositivo, não contempla a inexistência de risco adicional para o paciente ou outras pessoas, mas pode afetar a qualidade do diagnóstico informação (CDRH Ressonância Magnética Grupo de Trabalho 1997). Já um equipamento Compatível a RM, por outro lado, não é apenas RM segura, mas também pode ser usado no ambiente de RM com nenhum efeito significativo sobre o seu funcionamento ou sobre a qualidade da informação de diagnóstico. Procurar manter uma lista de equipamentos Seguro a MRI e Compatível a RM, incluindo restrições de utilização. Esta lista deve ser mantida em todos os centros de MR pelo oficial de segurança MRI. 
Trazer pacientes ambulatoriais ao ambiente de MRI usando uma cadeira de rodas ou maca apropriadas ao ambiente. Certifique-se de que nenhum tanque de oxigênio, sacos de areia, pedaço de metal, ou outros objetos ferromagnéticos estão escondidos sob cobertores ou lençóis ou guardado no equipamento de transporte.

Garantir que todos os indivíduos e pacientes que entram no ambiente de MRI não possuam objetos magnéticos em seus corpos, como por exemplo implantes, balas, estilhaços, grampos de cabelo, sutiãs, botões, zíperes, joias, ou ligados a seus corpos como piercings. Objetos magnéticos ou fixado nos corpos dos pacientes, familiares ou membros da equipe devem ser removidos, se possível, antes que as mesmas adentrem o ambiente de MRI. Todos os pacientes devem usar batas hospitalares e esvaziar seus bolsos e remover fechos metálicos para procedimentos de MRI. O vestuário regular dos pacientes pode conter objetos magnéticos que podem representar um perigo no ambiente de MRI. Revise-o.

O ACR [10] adota restrições para o acesso a todos os ambientes de ressonância magnética, por meio da aplicação do conceito de Quatro Zonas. Esse conceito prevê restrições progressivas no acesso ao ambiente de ressonância magnética:

- Zona I: Região do público em geral.

- Zona II: Região de acesso ou contato entre os funcionários com os pacientes e familiares.

- Zona III: Regiões de perigo, sala de espera próximo ao ambiente de MRI.

- Zona IV: Região da porta de acesso ao equipamento de MRI, como ilustra a Figura 1

Deve-se ter pessoal treinado para examinar todos os pacientes, oferecendo duas oportunidades distintas para eles responderem o questionário de triagem. Certifique-se que a equipe de MRI possui conhecimento completo sobre o histórico médico do paciente. Tenha em sua equipe uma pessoa especialmente treinada, para atuar como chefe de Segurança em MRI. Pelo menos anualmente, forneça a equipe treinamento em educação para a segurança em MRI. Tomar precauções para evitar queimaduras no paciente durante o procedimento.

Só use equipamentos e dispositivos, como extintores de incêndios, tanques de oxigênio, monitores fisiológicos e clipes de aneurisma, que foram testados e aprovados para uso em ambientes de ressonância magnética. Ter um plano de forma proativa para o gerenciamento de pacientes graves que necessitam de monitorizarão fisiológica e infusão contínua e na administração de drogas e gases. Proporcionar a todos os pacientes de ressonância magnética proteção auditiva, como tampões para 
os ouvidos. Nunca tente executar um código de parada cardiopulmonar ou ressuscitação dentro da Zona IV.

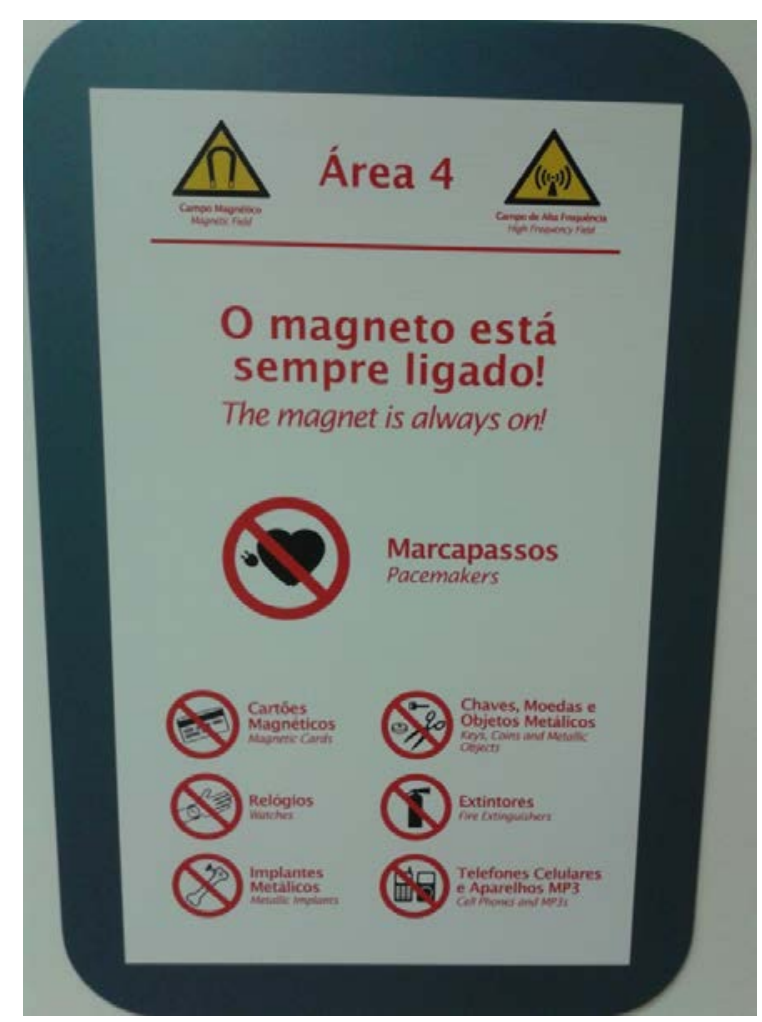

Figura 1: Sinalização na porta de uma sala de IRM indicando a zona e as restrições de acesso ao local.

\section{CONCLUSÕES}

O presente trabalho corrobora com a necessidade em ter estabelecidas políticas de segurança em MRI, com a importância dos procedimentos de triagem, com a educação continuada por parte de todos os envolvidos e a necessidade da criação de um novo cargo: Oficial de Segurança em Ressonância Magnética.

\section{AGRADECIMENTO}

A Coordenação de Aperfeiçoamento de Pessoal de Nível Superior (CAPES) pela bolsa de estudo no nível de Doutorado. 


\section{REFERÊNCIAS}

1. IBGE - Instituto Brasileiro de Geografia e Estatística. Estatísticas da saúde: Expectativa de vida do brasileiro aumenta para 74 anos. $<$ http://www.brasil.gov.br/noticias/arquivos/2012/11/29/expectativa-de-vida-do-brasileiroaumenta-para-74-anos. >. Último acesso: 23 Out. 2014

2. CNES - Secretaria de atenção à saúde.

<http://cnes.datasus.gov.br/Mod_Ind_Equipamento.asp?VEstado=35\&VMun=355030.> Último acesso: 12 Nov. 2014

3. Chen DW. Boy, 6, dies of skull injury during M.R.I. New York Times. July 31, 2001:B1, B5.

4. "U.S. Food and Drug Administration: FDA Safety Communication," <http://www.fda.gov/medicaldevices/safety/alertsandnotices/ucm135362.htm (2014).>. Último acesso: 23 Out. 2014

5. Two stuck to MRI machine for 4 hrs. Mumbai Mirror. http://www.mumbaimirror.com/mumbai/cover-story/Two-stuck-to-MRI-machine-for-4hrs/articleshow/45103043.cms. Último acesso: 16 Dez. 2014.

6. Man stabbed in eye during brain scan. The New Zealand Gerald. < http://www.nzherald.co.nz/nz/news/article.cfm?c_id=1\&objectid=11359290>. Último acesso: 16 Dez. 2014.

7. Chhattisgarh: Woman seriously injured after getting stuck in MRI machine. The Times Of India. $\quad<$ http://timesofindia.indiatimes.com/city/raipur/Chhattisgarh-Woman-seriously- 
injured-after-getting-stuck-in-MRI-machine/articleshow/42043632.cms>. Último acesso: 16 Dez. 2014.

8. "Institute for Magnetic Resonance Safety, Education, and Research:

Guidelines for Screening patients for MR producers and individuals for the MR environment. $<$ http://www.imrser.org/PaperPDFRecord.asp?WebRecID=82\&PgName=Guidelines\&WebRe cID=\&sb_SummaryTitle=\& (2014).>. Último acesso: 19 Nov. 2014

9. "ECRI Institute: Patient Death Illustrates the Importance of Adhering to Safety Precautions in Magnetic Resonance Environment,"

$<$ https://www.ecri.org/Documents/Patient_Safety_Center/hazard_MRI_080601.pdf>. Último acesso: 2 Dez. 2014

10. Expert Panel on MRS. Kanal E, Barkovich AJ, Bell C, et al. ACR guidance document on MR safe practices: 2013. J Magn Reson Imaging. 2013;37:501-530. 